
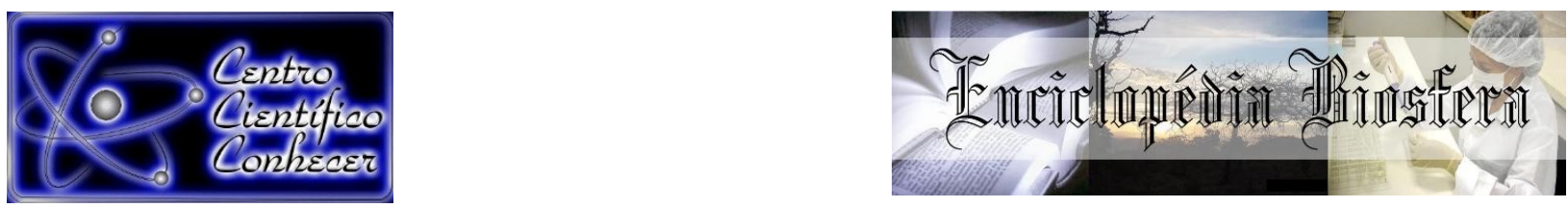

\title{
MANEJO DO SOLO COMO FONTE DE ALTERAÇÃO NA DINÂMICA DO CARBONO ORGÂNICO
}

Lucas de Sousa Oliveira ${ }^{1}$, Alfredo Mendonça de Sousa², José Israel Pinheiro", Valsérgio Barros da Silva ${ }^{3}$

${ }^{1}$ Bacharel em Agronomia, Doutorando em Ciências do Solo/Universidade Federal do Ceará (UFC), Fortaleza-CE, Brasil.

${ }^{2}$ Bacharel em Agronomia, Doutorando em Engenharia Agrícola/Universidade Federal do Ceará (UFC), Fortaleza-CE, Brasil. E-mail: alfredomendonca121@gmail.com.

${ }^{3}$ Bacharel em Agronomia, Doutorando em Engenharia Agrícola/Universidade Federal do Ceará (UFC), Fortaleza-CE, Brasil.

Recebido em: 15/02/2020 - Aprovado em: 15/03/2020 - Publicado em: 30/03/2020 DOI: $10.18677 /$ EnciBio_2020A14

RESUMO

A conversão dos ecossistemas naturais em áreas agrícolas, geralmente resulta em alterações na dinâmica do carbono do solo. Modificações nos estoques de carbono, pelo uso do solo, tem impactos sobre uma série de mudanças globais no planeta. Nesse sentido, destaca-se que a intervenção antrópica em ecossistemas naturais, por meio da exploração desordenada, tem conduzido a um processo de degradação ambiental significativo. Diante do exposto, objetivou-se avaliar o conteúdo de carbono orgânico do solo em área de mata secundária e de pastagem e sua relação com o estoque de carbono nesses ambientes. As amostras de solo foram coletadas em um Argissolo Vermelho Amarelo localizado no campus da Universidade Federal do Ceará-UFC, Fortaleza-CE, Brasil, nas camadas de $0-10 \mathrm{~cm}$ e $10-20 \mathrm{~cm}$ para determinação do conteúdo de carbono orgânico e na camada $0-20 \mathrm{~cm}$ para avaliação da quantidade desse elemento estocado no solo. A alteração do ecossistema natural ocasionou redução do conteúdo de carbono orgânico do solo na superfície e em profundidade, bem como menor estocagem de carbono orgânico no solo.

PALAVRAS-CHAVE: conservação do solo, mudanças climáticas, sequestro de carbono.

\section{SOIL MANAGEMENT AS A SOURCE OF CHANGE IN ORGANIC CARBON DYNAMICS}

\section{ABSTRACT}

The conversion of natural ecosystems into agricultural areas generally results in changes in the dynamics of soil carbon. Changes in carbon stocks, due to land use, have impacts on a series of global changes on the planet. In this sense, it is highlighted that anthropic intervention in natural ecosystems, through disordered exploration, has led to a process of significant environmental degradation. Given the above, the objective was to evaluate the organic carbon content of the soil in an area of secondary forest and pasture and its relationship with the carbon stock in these environments. The soil samples were collected in a Red Yellow Argisol located on the campus of the Federal University of Ceará-UFC, Fortaleza-CE, Brazil, in the 0-10 
$\mathrm{cm}$ and $10-20 \mathrm{~cm}$ layers to determine the organic carbon content and in the layer 0$20 \mathrm{~cm}$ to assess the amount of this element stored in the soil. The alteration of the natural ecosystem caused a reduction in the organic carbon content of the soil at the surface and in depth, as well as less storage of organic carbon in the soil.

KEY WORDS: soil conservation, climate change, carbon sequestration.

\section{INTRODUÇÃO}

Estima-se que o estoque de carbono global no solo é de aproximadamente $2.500 \mathrm{Gt}$, das quais, $70 \%$ são carbono orgânico e 30\% são de carbono inorgânico (LIU et al., 2020). De todo o carbono orgânico no solo, uma parte encontra-se na forma de matéria orgânica, sendo facilmente decomposta quando se realizam práticas de manejo não conservacionistas (KUMAR et al., 2020).

Quando ocorre a substituição de ecossistemas naturais por áreas com cultivos agrícolas, geralmente percebe-se o declínio no conteúdo de carbono orgânico no solo (COS). Estudos aventaram que essa redução está relacionada a fatores como o aumento dos processos erosivos, a aceleração dos processos de mineralização da matéria orgânica e oxidação de grande parte do carbono orgânico do solo e às menores quantidades de aportes orgânicos em sistemas manejados (TRAORÉ et al., 2019).

Dentre as principais estratégias de mitigação das causas antrópicas do efeito estufa destacam-se o menor uso de combustíveis fósseis, redução das taxas de desmatamento e de queima de material vegetal aportado pela vegetação, uso inadequado do solo e, mecanismos de maximização de sequestro de carbono orgânico (ZHENG et al., 2019).

No tocante ao carbono orgânico no solo, sabe-se que uma parte considerável se encontra na forma de matéria orgânica. No entanto, o material orgânico no solo pode ser facilmente decomposto quando se realizam práticas de manejo inadequado, contudo, é conhecido que o aumento no estoque de carbono é um processo dinâmico e lento, necessitando de práticas de manejo conservacionistas, principalmente, em regiões de clima tropical, onde a taxa de decomposição é mais acentuada devido às altas temperaturas e umidade do solo (SANTINI et al., 2019).

O conteúdo de COS resulta do equilíbrio entre a entrada (adição) e a decomposição (perda) do material orgânico, com influência direta dos componentes bióticos do sistema. As taxas de entrada e saída tem relação direta com a substituição da vegetação nativa por cultivos agrícolas, pela qualidade e quantidade de material vegetal aportado e pelo tipo de manejo empregado na área (PAUL, 2016). Logo, o uso do solo pode desenvolver um papel chave no conteúdo de COS.

Diante do exposto, o estudo parte da hipótese que alterações no uso do solo acarretam mudanças no conteúdo de COS. Objetivou-se com esse trabalho avaliar o conteúdo de conteúdo de carbono orgânico no solo (COS) em área de mata secundária e de pastagem e sua relação com o estoque de carbono orgânico nesses ambientes.

\section{MATERIAL E MÉTODOS}

A coleta das amostras de solo foi realizada no Campus do Pici da Universidade Federal do Ceará-UFC, Fortaleza-CE, Brasil, em um Argissolo Vermelho Amarelo (EMBRAPA, 2018). Para a determinação dos teores de matéria orgânica do solo foi adotado o delineamento inteiramente aleatorizado com parcelas subdivididas e sete repetições. Nas parcelas principais foram avaliados os dois tratamentos (mata secundária e área de pastagem), enquanto nas subparcelas os tratamentos avaliados foram as camadas $0-10$ e $10-20 \mathrm{~cm}$. Determinou-se o carbono orgânico 
pelo método de oxidação da matéria orgânica via úmida com dicromato de potássio em meio sulfúrico (WALKLEY; BLACK, 1934). A densidade do solo foi determinada a partir de amostras com estrutura preservada, coletadas em cilindros $\left(100 \mathrm{~cm}^{3}\right) \mathrm{e}$ secas a $105^{\circ} \mathrm{C}$, até massa constante (BLACKE; HARTGE, 1986).

Concernente à granulometria, a quantidade das frações areia, silte e argila são apresentadas na Tabela 1.

Tabela 1. Granulometria do solo em que foram coletadas as amostras.

\begin{tabular}{|c|c|c|}
\hline Areia & Silte & Argila \\
\hline - - - - - - - - - & $\mathrm{g} \mathrm{kg}$ & -- \\
\hline 84 & 9 & 7 \\
\hline
\end{tabular}

Os estoques de carbono orgânico do solo foram obtidos considerando a espessura da camada amostrada de $0-20 \mathrm{~cm}$, de acordo com a equação 1 :

$$
E s t C=\frac{C s * D s *\left(\frac{\text { Dref }}{D s} * e\right)}{10}
$$

(1)

em que,

EstC corresponde ao estoque de carbono orgânico em determinada profundidade $\left(\mathrm{Mg} \mathrm{ha}^{-1}\right)$, Cs é o teor de $\mathrm{C}$ orgânico total na profundidade amostrada $\left(\mathrm{g} \mathrm{kg}^{-1}\right), D$ s é a densidade do solo na profundidade amostrada $\left(\mathrm{kg} \mathrm{dm}^{-3}\right)$, Dref é a densidade do solo para a profundidade amostrada na área de referência e, "e" a espessura da camada considerada $(\mathrm{cm})$.

Os valores de densidade do solo obtidos foram 1,45 e $1,60 \mathrm{~g} \mathrm{~cm}^{-3}$ nas áreas de mata secundária e pastagem, respectivamente. Utilizou-se como valor de referência a densidade da área de mata secundária.

Os dados foram submetidos à análise de variância pelo teste $F$ e, quando significativos para este teste, comparou-se as médias utilizando-se o teste de Tukey $(p<0,05)$ para comparação entre as médias. Fez-se uso do software estatístico Sisvar (FERREIRA, 2013).

\section{RESULTADOS E DISCUSSÃO}

No que se refere aos teores de COS, houve diferença estatística entre as áreas avaliadas bem como nas profundidades amostradas (Tabela 2).

Tabela 2. Análise de variância para a variável teor de carbono orgânico em diferentes manejos e profundidades.

\begin{tabular}{ccccc}
\hline Fontes de Variação & GL & SQ & QM & F \\
\hline Áreas (A) & 1 & 2146,6 & 2146,6 & $265,1^{* *}$ \\
Resíduo & 12 & 97,1 & 8,1 & \\
\hline Parcelas & 13 & 2243,7 & & \\
\hline Profundidades (P) & 1 & 614,8 & 614,8 & $44,9^{* *}$ \\
Inter. A x P & 1 & 84,4 & 84,4 & $6,2^{*}$ \\
Resíduo & 12 & 164,2 & 13,7 & \\
\hline Total & 27 & 3107 & & \\
\hline CV\% - (A) & 11,6 & & & \\
CV\% - (P) & 15,1 & & & \\
\hline
\end{tabular}

** Significativo ao nível de $1 \%$ de probabilidade. * Significativo ao nível de $5 \%$ de probabilidade. 
O carbono orgânico do solo apresentou maiores valores na camada superficial (0-10 $\mathrm{cm}$ ), tanto na área de mata secundária como na de pastagem, no entanto, a área de mata secundária apresentou um maior conteúdo de carbono nas duas profundidades estudadas (0-10 e 10-20 cm) (Figura 1). De forma semelhante aos resultados encontrados por Rosa et al. (2014) e Eze et al. (2018) observaram que o teor de carbono orgânico diminuiu com a profundidade nas áreas avaliadas (Figura 1), o que é normal, uma vez que os maiores aportes de matéria orgânica são observados nas camadas superficiais.

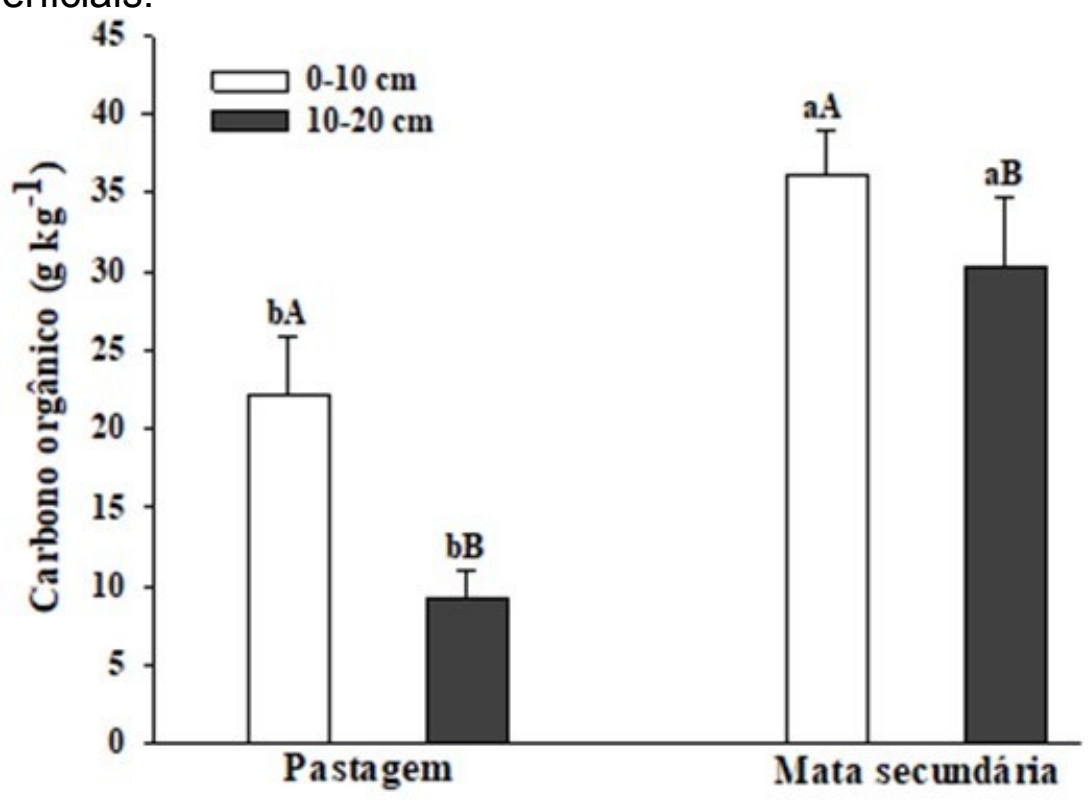

Figura 1. Valores médios de carbono orgânico $\left(\mathrm{g} \mathrm{kg}^{-1}\right)$ em diferentes áreas de um Argissolo Vermelho Amarelo nas profundidades de 0-10 cm e 10-20 cm. Médias seguidas pela mesma letra minúscula e/ou maiúscula não diferem estatisticamente entre si, entre e dentro das áreas analisadas, respectivamente, conforme o teste de Tukey $(p<0,05)$. Fonte: Autores

A manutenção e/ou melhoria da qualidade do solo em sistemas de cultivo é essencial para a produtividade agrícola e qualidade do ecossistema. Nesse sentido, o carbono orgânico do solo desempenha papel de destaque, sendo considerado um dos indicadores da qualidade do solo, servindo de base para a sustentabilidade do ambiente.

É importante salientar que o carbono constitui aproximadamente $58 \%$ da composição da matéria orgânica do solo. Apesar de representar uma fração pequena $(0,5$ a $5 \%)$ quando comparada à fração mineral do solo, a matéria orgânica é importante para os ecossistemas devido aos benefícios produzidos nas propriedades físicas, químicas e biológicas (SANTINI et al., 2019).

Segundo Oliveira Filho et al. (2019) em regiões tropicais a conversão de áreas florestais em áreas agrícolas aliado ao cultivo convencional, praticado durante anos consecutivos, é a principal causa da perda de carbono orgânico do solo, logo, essa redução no conteúdo de COS tem efeitos imediatos na capacidade de troca de cátions no meio e disponibilidade de nutrientes (BACCOT et al., 2020). Destaca-se que esses tipos de alterações nos ecossistemas naturais favorece o aumento na extensão de áreas descobertas, o que maximiza a perda de matéria orgânica e nutrientes por processos erosivos. Perdas de água e solo pela erosão hídrica diminuem a capacidade produtiva dos solos e favorece o aumento de áreas degradadas. 
No tocante ao estoque de carbono no solo verificou-se efeito significativo das áreas de manejos amostradas sobre a expressão desta variável (Tabela 3).

Tabela 3. Análise de variância para a variável estoque de carbono no solo sob mata nativa e pastagem.

\begin{tabular}{ccccc}
\hline Fontes de variação & $\mathrm{GL}$ & $\mathrm{SQ}$ & $\mathrm{QM}$ & $\mathrm{F}$ \\
\hline Áreas & 1 & 6702,1 & 6702,1 & $47,9^{\star *}$ \\
Resíduo & 28 & 3913 & 139,8 & \\
Total & 29 & & & \\
\hline CV\% & 21,2 & & & \\
\hline
\end{tabular}

** Significativo ao nível de $1 \%$ de probabilidade.

Verifica-se menor estoque na área de pastagem (Figura 2). Rosa et al. (2014), em seu trabalho, salientaram o fato de que a medida que ocorre a conversão de um sistema natural em pastagem ocorre alterações no carbono estocado no solo, uma vez que, o carbono acaba sendo perdido depois do desmatamento da área, queima e intervenções frequentes no preparo do solo.

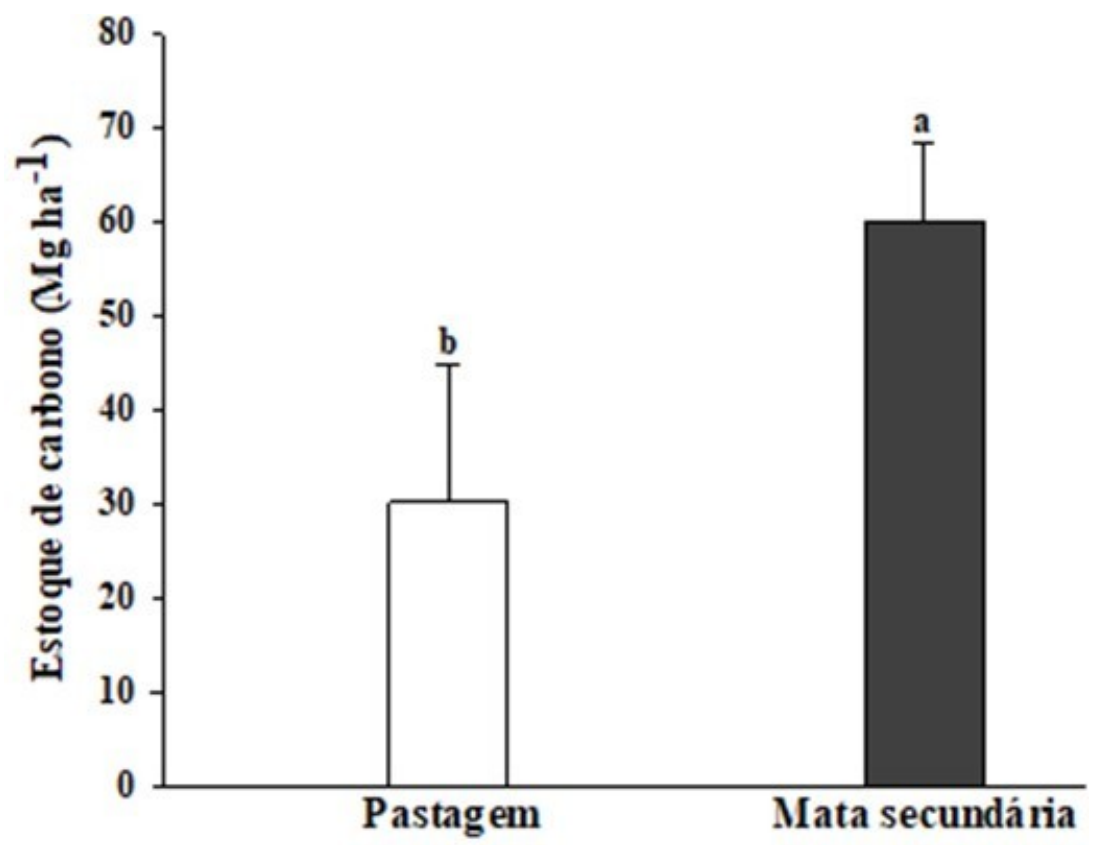

Figura 2. Valores médios de estoque de carbono no solo sob diferentes manejos. Médias seguidas pela mesma letra minúscula não diferem estatisticamente entre si pelo teste de Tukey $(p<0,05)$. Fonte: Autores

Cerri et al. (2006), em seu trabalho, salientaram o fato de que após a mudança de uso da terra, a capacidade de recuperação do solo pode ser entre 50 e $75 \%$ da quantidade de COS inicialmente existente, entretanto, esse potencial de recuperação está diretamente associado à fatores ambientais, de uso da área e manejo adotado.

O carbono orgânico do solo (COS) contribui de forma determinante nas melhorias observadas em atributos físicos, químicos e biológicos do solo (LIU et al., 2020). Logo, o COS além de fonte de nutrientes para a microbiota do solo, atua no processo de armazenamento e liberação de nutrientes na solução do solo, disponibiliza água para os vegetais e organismos e contribui na formação e 
estabilização dos agregados. Desse modo, sistemas de manejo que favoreçam a conservação e incremento do COS são desejáveis para mitigar as emissões de $\mathrm{CO}_{2}$ para atmosfera.

Segundo Kumar et al. (2020) recomenda-se o uso de gramíneas forrageiras para estocar e distribuir o carbono na superfície do solo, tanto pela elevada entrada de biomassa quanto por contribuir para diminuição no revolvimento do solo nessas áreas. No entanto, a conversão de florestas em áreas de pastagem causa alterações na dinâmica do ecossistema manejado, e ressalta-se que, mesmo bem manejada, a contribuição pela manutenção da floresta não é somente pelo sequestro de carbono, mas pela manutenção dos estoques nessas áreas, reduzindo a emissão de gases oriundos da degradação acelerada ou queima da biomassa vegetal.

É de interesse da comunidade científica mundial o conhecimento do estoque de carbono e sua dinâmica no solo em sistemas naturais e manejados, uma vez que, o melhor entendimento se torna importante no desenvolvimento de novas tecnologias para a implantação de sistemas sustentáveis, bem como reduzir as emissões $\mathrm{CO}_{2}$ para atmosfera.

\section{CONCLUSÃO}

A alteração do ecossistema natural ocasionou redução do conteúdo de carbono orgânico do solo na superfície e em profundidade bem como menor estocagem de carbono no solo.

\section{REFERÊNCIAS}

BACCOT, C.; PALLIER, V.; THOM, M. T.; THURET-BENOIST, H.; FEUILLADECATHALIFAUD, G. Valorization of extracted organic matter from municipal solid waste leachate: Application to soils from France and Togo. Waste Management, v. 102, n. 1, p. 161-169, 2020. Disponível em: <https://www.ncbi.nlm.nih.gov/pubmed/31678802>. DOI: 10.1016/j.wasman.2019.10.040.

BLAKE, G. R.; HARTGE, K. H. Particle density. In: Klute. A. (ed). Methods of soil analysis. 2. ed. Madison, America Society of Agronomy, Soil Science Society of America, pt. 1, p. 377-382, 1986.

CERRI, C. C.; BERNOUX, M.; CERRI, C. E. P.; LAL, R. Challenges and opportunities of soil carbon sequestration in Latin America. In: LAL, R.; CERRI, C. C.; BERNOUX, M.; ETCHEVERS, J. D.; CERRI, C. E. P. (Ed). Carbon sequestration in soils of Latin America. New York: The Haworth Press, 2006. v.1, p. 41-47.

EMPRESA BRASILEIRA DE PESQUISA AGROPECUÁRIA - EMBRAPA. Centro Nacional de Pesquisa de Solos. Sistema Brasileiro de Classificação de Solos. $5^{\text {a }}$ ed. Rio de Janeiro: Embrapa Solos. 353p. 2018.

EZE, S.; PALMER, S. M.; CHAPMAN, P. J. Soil organic carbon stock in grasslands: Effects of inorganics fertilizers, liming and grazing in different climate settings. Journal of Environmental Management, v. 223, p. 74-84, 2018. Disponível em: < https://www.sciencedirect.com/science/article/pii/S0301479718306558>.

DOI: doi.org/10.1016/j.jenvman.2018.06.013.

FERREIRA, D. F. Sistema de análise de variância. Versao 5.6. Lavras: UFLA, 2013. 
KUMAR, R.; BHATNAGAR, P. R.; KAKADE, V.; DOBHAL, S. Tree plantation and soil water conservation enhances climate resilience and carbon sequestration of agro ecosystem in semi-arid degraded revine lands. Agricultural and Forest Meteorology, v. 282-283, n. 107857, 2020. Disponível em: < https://www.sciencedirect.com/science/article/pii/S0168192319304733>. DOI: doi.org/10.1016/j.agrformet.2019.107857.

LIU, X.; CHEN, D.; YANG, T.; HUANG, F.; FU, S.; LI, L. Changes in soil labile and recalcitrant carbon pools after land-use change in a semi-arid agro-pastoral ecotone in Central Asia. Ecological Indicators, v. 110, n. 105925, 2020. Disponível em: < https://www.sciencedirect.com/science/article/abs/pii/S1470160X19309203>. DOI: doi.org/10.1016/j.ecolind.2019.105925.

OLIVEIRA FILHO, J. DE S.; VIEIRA, J. N.; SILVA, E. M. R.; OLIVEIRA, J. G. B.; PEREIRA, M. G.; BRASILEIRO, F.G. Assessing the effects of 17 years of grazing exclusion in degraded semi-arid soils: Evaluation of soil fertility, nutrients pools and stoichiometry. Journal of Arid Environments, v. 166, p. 1-10, 2019.

PAUL, E. A. The nature and dynamics of soil organic matter: Plant inputs, microbial transformations, and organic matter stabilization. Soil Biology \& Biochemistry, v. 98, p. 109-126, 2016. Disponível em: < https://www.sciencedirect.com/science/article/abs/pii/S0038071716300281>. DOI: doi.org/10.1016/j.soilbio.2016.04.001

ROSA, R.; SANO, E. E.; ROSENDO, J. S. Estoque de carbono em solos sob pastagens cultivadas na Bacia hidrográfica do Rio Parnaíba. Sociedade \& Natureza, v. 26, n. 2, p. 333-351, 2014. Disponível em: < http://www.scielo.br/scielo.php?script=sci_arttext\&pid=S1982-45132014000200333 >. DOI: doi.org/10.1590/1982-451320140210

SANTINI, N. S.; ADAME, M. F.; NOLAN, R. H.; MIGUELAJAUREGUI, Y.; PIÑERO, D.; MASTRETTA-YANES, A.; CUERVO-ROBAYO, A. P.; EAMUS, D. Storage of organic carbon in the soils of Mexican temperate forests. Forest Ecology and Management, v. 446, p. 115-125, 2019. Disponível em: < https://www.sciencedirect.com/science/article/abs/pii/S0378112719302853>. DOI: doi.org/10.1016/j.foreco.2019.05.029

TRAORÉ, S.; OUATTARA, K.; ILSTEDT, U.; SCHMIDT, M.; THIOMBIANO, A.; MALMER, A.; NYBERG, G. Effect of land degradation on carbon and nitrogen pools in two soil types of a semi-arid landscape in West Africa. Geoderma, v. 241242, p. 330-338, 2015. Disponível em< https://www.sciencedirect.com/science/article/pii/S001670611400425X>. $\quad$ DOI: doi.org/10.1016/j.geoderma.2014.11.027

WALKLEY, A.; BLACK, I. A. An examination of Degtjareff method for determining soil organic matter, and proposed modification of the chromic acid tritation method. Soil Science, v. 37, n. 1, p. 29-38, 1934. DOI: http://dx.doi.org/10.1097/00010694193401000-00003 
ZHENG, X.; STREIMIKIENE, D.; BALEZENTIS, T.; MARDANI, A.; CAVALLARO, $\mathrm{F}$.; LIAO, $\mathrm{H}$. A rewiew of greenhouse gas emission profiles, dynamics, and climate change mitigation efforts across the key climate change players. Journal of Cleaner Production, v. 234, p. 1113-1133, 2019. Disponível em: < https://www.sciencedirect.com/science/article/pii/S0959652619320943>. DOI: doi.org/10.1016/j.jclepro.2019.06.140 